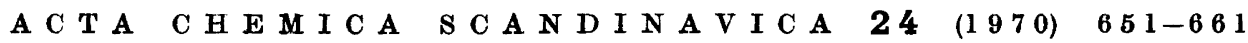

\title{
Intramolecular Exchange Rates from Complex NMR Spectra
}

\author{
KJELL-IVAR DAHLQVIST And STURE FORSEN \\ Division of Physical Chemistry, Lund Institute of Technology Chemical Center, \\ Box 740, S-220 07 Lund 7, Sweden
}

T OR B J ö R N A L M

Autocode AB, S.171 49 Solna, Sweden

\begin{abstract}
Equations for the lineshape in strongly coupled NMR spectra of molecular systems undergoing intramolecular chemical exchange have been evaluated according to the density matrix theory of Kaplan and Alexander, and a computer programme has been developed by means of which the lineshape equations may be solved for up to four-spin systems. Calculated NMR spectra at different exchange rates in $\mathrm{ABCD} \rightarrow \mathrm{AA}^{\prime} \mathrm{BB}^{\prime}, \mathrm{AA}^{\prime} \mathbf{B B}^{\prime} \rightarrow \mathrm{A}_{4}, \mathrm{ABC} \rightarrow \mathrm{A}_{2} \mathrm{~B}$, and other types of exchanging systems are presented and discussed. The validity of approximate formulae and simplified procedures for the evaluation of rate parameters from complex NMR spectra is also briefly discussed.
\end{abstract}

Tn the study of restricted internal rotation and interconversion of molecular

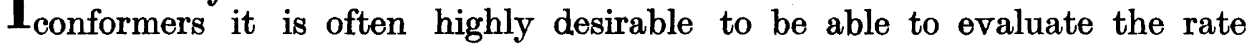
parameters from NMR spectra of spin systems of more than two spins. For example, in the limit of very slow inversion rate of the nitrogen atom in $N$-methyl aziridine, the ring protons give rise to a complex $\mathrm{AA}^{\prime} \mathrm{BB}^{\prime}$-type spectrum which in the fast inversion limit is converted into an $\mathbf{A}_{\mathbf{4}}$-type spectrum consisting of a single resonance line. A second example is provided by parasubstituted benzaldehydes. As shown by Anet and Ahmad ${ }^{1}$ the rotation of the aldehyde group is restricted and the ring proton spectrum is transformed from an $\mathrm{ABCD}$-type spectrum at low rates of rotation to an $\mathrm{AA}^{\prime} \mathrm{BB}^{\prime}$-type spectrum at high interconversion' rates. A large number of other examples is given in the excellent and comprehensive review by Binsch. ${ }^{2}$

When a complex NMR spectrum has been encountered in the course of a kinetic study, the stratagem usually employed is to reduce the complexity by means of isotopic substitution (for example exchanging $H$ for $D$ ) or by substitution of a hydrogen atom with a methyl group, a halogen atom, etc. These 
methods of simplifying spectra have been used in the investigation of interconversion processes in some dioxanes, ${ }^{3}$ in benzofuroxans, ${ }^{4}$ and in cyclohexane. ${ }^{5}$

Isotopic substitution is, however, often rather laborious and time-consuming and, in order to be fully exploited, in addition commonly requires the application of double irradiation techniques. Non-isotopic substituents may have undesirable steric and/or electronic side effects on the process to be investigated which are difficult to disentangle when the physical significance of the kinetic parameters is considered. Sometimes simple first order or ABtype lineshape equations have also been used for the evaluation of rate parameters in spectra of higher degree of complexity than for which these equations are derived. ${ }^{6-8}$

Much is to be gained if the influence of rate processes on the lineshape in complex spin systems could be calculated. In the present work we describe the application of the theory of Kaplan ${ }^{8,10}$ and Alexander ${ }^{11,12}$ in the development of a computer programme for calculations of the lineshape in NMR spectra of strongly coupled spin system of up to four nuclei undergoing intramolecular chemical exchange.

\section{THEORETICAL BACKGROUND OF THE COMPUTER PROGRAMME}

In the absence of chemical exchange the equation of motion of the density matrix $\varrho$ of a system of nuclear spins may be written

$$
\frac{\mathrm{d} \varrho}{\mathrm{d} t}=\frac{i}{\hbar}[\varrho, H]+\Gamma(\varrho)
$$

where the brackets in the first term on the right hand side denote the commutator of the spin density matrix and the Hamiltonian of the spin system in the presence of a static magnetic field and a weak observing r.f. field. The term $\Gamma(\varrho)$ in eqn. (1) is added in order to take relaxation processes into account. In the following we shall restrict our treatment to weak $H_{1}$ fields and the relaxation term will be replaced by a simple damping term $\varrho / T_{2}$ - equal for all off-diagonal elements of the density matrix in the energy representation.

According to Kaplan ${ }^{9,10}$ eqn. (1) may be generalized to take intramolecular exchange or rearrangements into account by adding to the right hand of the equation an additional term $(\mathrm{R} \varrho \mathrm{R} \varrho-\varrho) / \tau$, where $\mathrm{R}$ is an exchange operator to be further discussed below, and $\tau^{-1}$ is the probability of an exchange event. The complete equation of motion of $\varrho$ in the presence of chemical exchange then becomes

$$
\frac{\mathrm{d} \varrho}{\mathrm{d} t}=\frac{\mathrm{i}}{\hbar}[\varrho, H]-\frac{\varrho}{T_{2}}+\frac{\mathrm{R} \varrho \mathrm{R}-\varrho}{\tau}
$$

The exchange operator $\mathbf{R}$ is defined in such a way that if $\psi$ is the wave function of the spin system before an exchange then the wave function after an exchange event is $\mathbf{R} \psi$, thus

$$
\psi \rightarrow \mathbf{R} \psi
$$

The density matrix after an exchange event is obtained by a unitary transformation 


$$
\varrho \rightarrow \mathbf{R}_{\varrho} \mathbf{R}^{-1}=\mathbf{R} \varrho \mathbf{R}
$$

The equality in eqn. (4) is valid because $R^{2}=1$ - a second exchange event will restore the wave function $\mathbf{R} \psi$ to the original wave function $\psi$.

As basis functions for the representation of $\psi$ we will use simple products of the spin functions of the individual nuclei $i$

$$
\psi_{k}=\prod_{\mathrm{i}} u\left(I^{\mathrm{i}}, m_{\mathrm{i}}\right)
$$

The nature of the exchange operator $R$ in eqns. (2) to (4) may be illustrated for the case of intramolecular exchange in a four-spin system. The basis product function may be written as a column matrix

$$
\psi=\left|\begin{array}{llll}
\alpha & \alpha & \alpha & \alpha \\
\alpha & \alpha & \alpha & \beta \\
\alpha & \alpha & \beta & \alpha \\
\alpha & \beta & \alpha & \alpha \\
\beta & \alpha & \alpha & \alpha \\
\alpha & \alpha & \beta & \beta \\
\alpha & \beta & \alpha & \beta \\
\beta & \alpha & \alpha & \beta \\
\beta & \alpha & \beta & \alpha \\
\beta & \beta & \alpha & \alpha \\
\alpha & \beta & \beta & \alpha \\
\alpha & \beta & \beta & \beta \\
\beta & \alpha & \beta & \beta \\
\beta & \beta & \alpha & \beta \\
\beta & \beta & \beta & \alpha \\
\beta & \beta & \beta & \beta
\end{array}\right|
$$

where $\alpha \beta \beta \alpha$ etc. are shorthand notations for $\alpha(1) \beta(2) \beta(3) \alpha(4)$ etc. For an exchange process in which nucleus 1 is exchanged with nucleus 2 and nucleus 3 with nucleus 4 the exchange matrix $R$ becomes

$$
R=\left|\begin{array}{lllllllll}
1 & & & & & & & \\
1 & & & & & & \\
1 & 1 & & & & & \\
& 1 & 1 & & & & \\
& & 1 & 1 & & & \\
& & 1 & 1 & 1 & & \\
& & & 1 & & & & \\
& & & & & 1 & 1 & \\
& & & & & & 1 & 1
\end{array}\right|
$$

and the spin wave function after the exchange becomes

$$
\mathbf{R}_{\psi}=\left|\begin{array}{llll}
\alpha & \alpha & \alpha & \alpha \\
\alpha & \alpha & \beta & \alpha \\
\alpha & \alpha & \alpha & \beta \\
\beta & \alpha & \alpha & \alpha \\
\alpha & \beta & \alpha & \alpha \\
\alpha & \alpha & \beta & \beta \\
\beta & \alpha & \beta & \alpha \\
\alpha & \beta & \beta & \alpha \\
\alpha & \beta & \alpha & \beta \\
\beta & \beta & \alpha & \alpha \\
\beta & \alpha & \alpha & \beta \\
\beta & \alpha & \beta & \beta \\
\alpha & \beta & \beta & \beta \\
\beta & \beta & \beta & \alpha \\
\beta & \beta & \alpha & \beta \\
\beta & \beta & \beta & \beta
\end{array}\right|
$$

Acta Chem. Scand. 24 (1970) No. 2 
The complete spin Hamiltonian, in a frame of reference rotating with the angular frequency $\omega$ of the r.f. field expressed in angular frequency units, may be written

$$
H=\sum_{\mathbf{i}} I_{x}^{\mathrm{i}}\left(\omega_{\mathrm{i}}-\omega\right)+2 \pi \sum_{\mathbf{i}<\mathrm{j}} J_{\mathrm{ij}} I^{\mathrm{i}} I^{\mathrm{j}}+\sum_{\mathrm{i}} \gamma^{\mathrm{i}} I_{x}^{\mathrm{i}} H_{1}
$$

with the $H_{0}$ field along the negative $z$-axis and the $H_{1}$ field along the negative $x$-axis and

$$
\omega_{i}=\gamma^{i} \hbar H_{0}\left(1-\sigma^{i}\right)
$$

where $\sigma^{i}$ is the screening constant of nucleus $i$.

The component of the resulting angular momentum in the $z$-direction is

$$
\mathrm{F}_{z}=\sum_{\mathrm{i}} I_{z} \mathbf{i}
$$

If the observing $\mathrm{H}_{1}$ field is weak, transitions between two spin states $k$ and $l$ will be observed only if

$$
\mathrm{M}_{l}=\mathrm{M}_{k} \pm \mathbf{l}
$$

where $M$ represents the eigenvalues of the operator $F_{x}$ defined in (8). If this selection rule is combined with eqns. (2) and (6) one obtains the following expression for the elements of the density matrix

$$
\begin{gathered}
\left.\frac{\mathrm{d} \varrho_{k l}}{\mathrm{~d} t}=\frac{1}{\tau} \sum_{n, m} \mathrm{R}_{k n} \varrho_{n m} \mathrm{R}_{m l}-\varrho_{k l}\right]-\frac{\varrho_{l k}}{T_{2}}- \\
-\mathrm{i} \cdot \varrho_{k l}\left[\sum_{\mathrm{i}}\left(\omega_{\mathrm{i}}-\omega\right)\left[\left(I_{z}^{\mathrm{i}}\right)_{k k}-\left(I_{x}^{\mathrm{i}}\right)_{l}\right]+\sum_{\mathrm{i}<\mathrm{j}} J_{\mathrm{ij}}\left[\left(I_{x}^{\mathrm{i}} I_{x}^{\mathrm{j}}\right)_{k k}-\left(I_{z}^{\mathrm{i}} I_{z}^{\mathrm{j}}\right)_{l l}\right]+\right. \\
+\frac{\mathrm{i}}{2} \sum_{\mathrm{i}<\mathrm{j}} J_{\mathrm{ij}}\left[\varrho, I^{\mathrm{i}}+I_{-}^{\mathrm{j}}+I_{-}^{\mathrm{i}} I_{+}^{\mathrm{j}}\right]_{k l}+\mathrm{i} \omega_{r}\left(\varrho_{k k}-\varrho_{u}\right) \sum_{\mathrm{i}}\left(\mathrm{I}_{x}^{\mathrm{i}}\right)_{k l}
\end{gathered}
$$

where $I_{ \pm}^{\mathrm{j}}=I_{x}^{\mathrm{j}} \pm \mathrm{i} I_{y}^{\mathrm{j}}$, and $\omega_{r}=\gamma H_{1}$. When $I_{ \pm}$operates on a spin function $U(I, m)$ the function is transformed into $U(I, m \pm 1)$. Consequently, when the operator $I_{+}{ }^{i} j_{-}+I_{-}^{i} I_{+}^{j}$ acts on a basis spin function according to eqn. (5) the result will be zero if $m_{\mathrm{i}}=m_{\mathrm{j}}$ and if $m_{\mathrm{i}} \neq m_{\mathrm{j}}$ the spin functions of nuclei $\mathrm{i}$ and $\mathrm{j}$ are permuted.

The set of simultaneous equations described by eqn. (10) may be solved numerically on an electronic computer. To obtain the spectral lineshape under the quasi-stationary conditions of slow passage, all time derivatives $\mathrm{d} \varrho_{k l} / \mathrm{d} t$ on the left hand side of the equation may be set equal to zero.

In the density matrix formalism the ensemble average of the expectation value of an operator $Q$ is given by

$$
\langle\mathbf{Q}\rangle=\sum_{\mathrm{i}, \mathrm{j}} \varrho_{\mathrm{ji}} \mathbf{Q}_{\mathrm{ij}}=\operatorname{Tr}(\varrho \mathbf{Q})
$$


where Tr denotes the trace of the product matrix $\varrho$ Q. Eqn. (11) may be applied to obtain the intensity of the absorption spectrum $I(\omega)$ which is proportional to the expectation value of $I_{y}$ in the rotating frame

$$
I(\omega)=\operatorname{Im} \operatorname{Tr}\left(\varrho_{k l}\left(\sum_{\mathbf{i}} I_{+}{ }^{\mathfrak{i}}\right)_{l k}\right)
$$

where Im denotes the imaginary part.

From eqns. (10) and (12) the lineshape in NMR spectra of strongly coupled spin systems undergoing intramolecular exchange processes may be calculated. A Fortran IV programme named DENSMAT based on eqns. (10) and (12) has been developed and applied to a number of practical problems. ${ }^{13,14}$ Even on a large and fast computer a complete lineshape calculation for a complex spin system requires considerable computation time. In order to obtain a good reproduction of the lineshape for a given set of spin coupling constants, chemical shifts and rate parameters it is often necessary to solve eqns. (10) and (12) at relatively closely spaced frequency intervals - from several hundred to a thousand points on the lineshape curve may be required.

The present programme allows calculation of lineshapes in up to fourspin systems but can easily be extended to larger spin systems. The theoretical absorption intensities may either be obtained in printed or plotted form.

The following types of intramolecular exchange processes may be treated by means of the present programme:

\section{Slow exchange limit}

$\mathrm{AB}$

$\mathrm{ABC}$ or $\mathrm{ABX}$

$\mathrm{AA}^{\prime} \mathrm{BB}^{\prime}$

$\mathrm{ABCD}$

\section{Fast exchange limit}

$\mathrm{A}_{2}$

$A_{2} B$ or $A_{2} X$

$A_{4}$

$\mathrm{AA}^{\prime} \mathrm{BB}^{\prime}$

The notation above requires some comment. The notation refers only to the effective type of spin systems obtained and does not indicate which nuclei are exchanging. Thus in the case of $A B C \rightarrow A_{2} B$ the nuclei designated by $A$ and $B$ in the slow exchange limit may for example be considered to undergo intramolecular exchange; in the case $\mathrm{AA}^{\prime} \mathrm{BB}^{\prime} \rightarrow \mathrm{A}_{4}, \mathrm{~A}$ is exchanged with $\mathrm{B}$ and $\mathrm{A}^{\prime}$ with $\mathrm{B}^{\prime}$, and finally in the case $\mathrm{ABCD} \rightarrow \mathrm{AA}^{\prime} \mathrm{BB}^{\prime}$, nucleus $\mathrm{A}$ may undergo exchange with $\mathrm{B}$ and $\mathrm{C}$ with $\mathrm{D}$.

The dependence of the lineshape on the rate of exchange in some of the above types of systems will be illustrated below. The calculations in the present paper have in part been performed on a CDC 3600 computer and in part on an IBM 360/75 computer. For a given set of starting parameters each point in the spectrum of a three-spin system requires $c a .0 .07$ sec on the CD 3600 computer and $c a$. $0.012 \mathrm{sec}$ on the IBM 360/75 computer. For a four-spin system the corresponding figures are $c a$. $1.7 \mathrm{sec}$ and $0.29 \mathrm{sec}$, respectively. Further details of the programme may be obtained directly from the authors. 


\section{APPLICATIONS TO COMMON TYPES OF INTRAMOLECULAR EXCHANGE PROCESSES}

A) Exchange in two-spin systems. The $\mathrm{AB} \rightarrow \mathrm{A}_{2}$ type of exchange process is quite common in certain conformational equilibria, for example when protons on a methylene group which are nonequivalent at slow interconversion rates become effectively chemically equivalent at higher exchange rates. Analytical expressions for the lineshape in this type of exchange have been derived by Alexander ${ }^{11,12}$ ( $c f$. also Binsch ${ }^{2}$ ) and applied to a number of investigations and thus this case will only be briefly commented upon here.

From the equations by Alexander, Beauchamp ( $c f$. Ref. 54 in Ref. 2) derived a closed expression valid in the slow exchange region for the width at half height $\left(\Delta v_{1 / 2}\right)$ of the signals in the $\mathrm{AB}$ quartet:

$$
\pi \Delta v_{1 / 2}=\frac{1 \pm J_{\mathrm{AB}}\left[\left(\nu_{\mathrm{A}}-v_{\mathrm{B}}\right)^{2}+J_{\mathrm{AB}}^{2}\right]^{1 / 2}}{\tau}+\frac{1}{T_{2}}
$$

where $v_{\mathrm{A}}$ and $\nu_{\mathrm{B}}$ are the chemical shifts of nuclei $\mathrm{A}$ and $\mathrm{B}$ and $J_{\mathrm{AB}}$ is the spinspin coupling constant. The plus sign in eqn. (13) applies to the outer lines and the minus sign to the inner lines.

An expression for the calculation of the exchange rate $\tau_{\mathrm{c}}$ at the point of coalescence with the assumption that $\mathrm{I} / \boldsymbol{T}_{2}=0$ has been derived by Kurland et $a l .:^{15}$

$$
\tau_{\mathrm{C}}=\frac{2}{\pi}\left[\left(\nu_{\mathrm{A}}-v_{\mathrm{B}}\right)^{2}+6 J_{\mathrm{AB}}^{2}\right]^{-1 / 2}
$$

Although these equations can be helpful for a preliminary evaluation of exchange rates, recourse to the complete lineshape equations is recommended for an accurate analysis.

B) Exchange in three-spin systems. The effect of chemical exchange of nuclei $A$ and $B$ on the lineshape in a moderately strongly coupled ABC system is shown in Fig. 1 (the parameter set employed is listed in the figure caption). As is evident from Fig. 1 two lines remain essentially unbroadened by the exchange. To understand this we may temporarily assume that the nonexchanging proton can be treated in the $\mathrm{X}$ approximation. The two sharp lines that correspond to transitions of nucleus $\mathbf{X}$ between those energy levels in which the two exchanging nuclei are in the same spin states $(\alpha \alpha \beta \rightarrow \alpha \alpha \alpha$ or $\beta \beta \beta \rightarrow \beta \beta \alpha)$ regardless of whether the spectrum is of type $\mathrm{ABX}$ or $\mathrm{A}_{2} \mathrm{X}$. Obviously a spin exchange of nuclei $A$ and $B$ in an $A B X$ spin system will leave the spin states $\alpha(\mathrm{A}) \alpha(\mathrm{B})$ and $\beta(\mathrm{A}) \beta(\mathrm{B})$ unchanged. In a true $\mathrm{ABX}$ spectrum therefore the two $X$ transitions involving these states would be completely unaffected by the intramolecular exchange process. The same type of reasoning applies also, although less rigorously, to moderately strongly coupled, or rather ABK-type, three-spin systems where $A$ and $B$ undergo exchange, as is illustrated by Fig. 1.

The appearance of two essentially unbroadened lines in some three-spin systems as described above can be very useful for distinguishing between inter-and intra-molecular exchange of the A and B nuclei in the ABX or ABK case. As long as only intramolecular exchange occurs, the outer lines in 


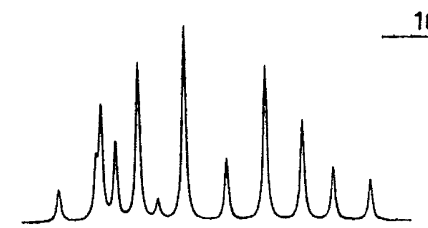

$\tau=100.0 \mathrm{sec}$

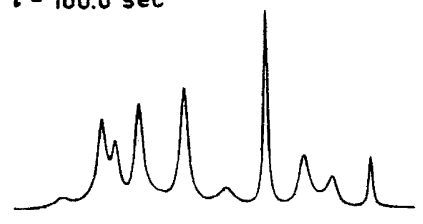

$r=0.400 \mathrm{sec}$

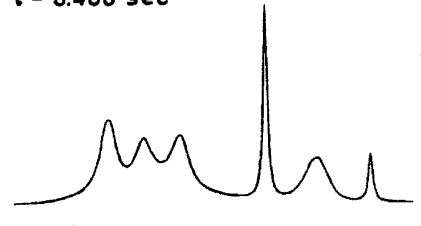

$\tau=0.100 \mathrm{sec}$

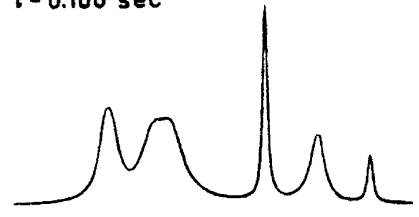

$\tau=0.045 \mathrm{sec}$

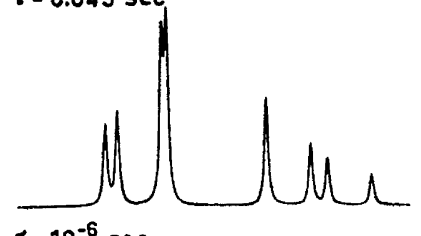

$r=10^{-6} \mathrm{sec}$

Fig. 1. Examples of theoretically calculated three-spin spectra of ABK-type which are converted to an $A_{2} K$ spectrum by means of fast interchange between $A$ and $B$. The spectra have been calculated with the following values for chemical shifts and spin coupling constants $\nu_{\mathrm{A}}=0.0 \mathrm{~Hz} ; v_{\mathrm{B}}=$ $5.0 \mathrm{~Hz} ; \nu_{\mathrm{C}}=15.0 \mathrm{~Hz} ; J_{\mathrm{AB}}=3.0 \mathrm{~Hz} ; J_{\mathrm{AK}}=$ $3.0 \mathrm{~Hz} ; J_{\mathrm{BK}}=3.0 \mathrm{~Hz}$. A spin-spin relaxation time $T_{2}$ of 1 sec has been used for these calculations.
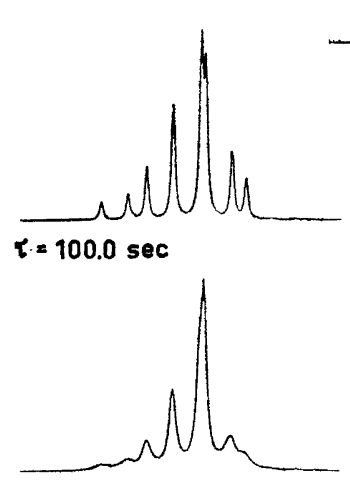

$\tau=0.400 \mathrm{sec}$

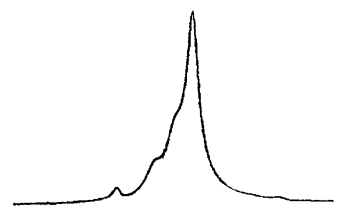

$\tau=0.045 \mathrm{sec}$
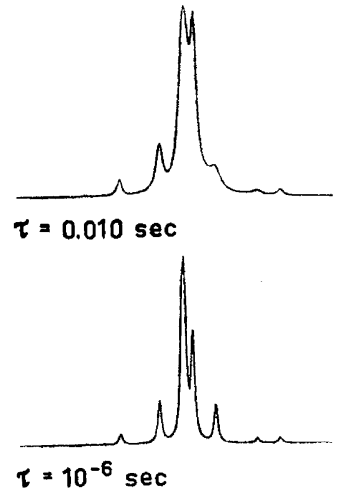

Fig. 2. Examples of theoretical three-spin spectra of ABC-type which are converted to an $A_{2} B$ spectrum by fast interchange between $A$ and $B$. These spectra have been calculated with $v_{\mathrm{A}}=0.0 \mathrm{~Hz} ; v_{\mathrm{B}}=5.0 \mathrm{~Hz}$; $v_{\mathrm{C}}=6.0 \mathrm{~Hz} ; J_{\mathrm{AB}}=3.0 \mathrm{~Hz} ; J_{\mathrm{AC}}=3.0 \mathrm{~Hz}$; $J_{\mathrm{BC}}=5.0 \mathrm{~Hz}$, and $T_{2}=1.0 \mathrm{sec}$.

the $\mathrm{X}$ or $\mathrm{K}$ part will remain sharp. However, if intermolecular exchange of the $A$ and $B$ nuclei reaches such a rate that it effects the NMR spectra even these $\mathrm{X}$ or $\mathrm{K}$ lines will become broadened. A molecule in which this effect can be observed is ${ }^{15} \mathrm{~N}$-formamide. The PMR spectrum of this molecule at slow inter-

Acta Chem. Scand. 24 (1970) No. 2 
conversion rates consists of two independent ABK spectra displaced with the ${ }^{15} \mathrm{~N}-\mathrm{H}$ spin couplings. ${ }^{16}$ The rotation around the $=\mathrm{C}-\mathrm{N}-$ bond re-

presents the intramolecular exchange, and the chemical exchange of the NH protons represents the intermolecular exchange process. In principle it is possible to evaluate the rates of both the intramolecular and the intermolecular exchange from the NMR spectra.

A second important consequence of the appearance of lines in the ABK or ABX case unaffected by intramolecular exchange processes is that the line width of the unbroadened signals may be used for estimates of $T_{2}$ of the nuclei in the molecule undergoing exchange. In this way it may in certain cases prove possible to obtain more reliable values of the intramolecular exchange rate from lineshape analysis in the region of slow and fast exchange where the exchange broadening of spectral lines is small.

In strongly coupled ABC spectra intramolecular exchange of two nuclei affects all lines in the spectra as shown in Fig. 2.

In three-spin systems approaching the $\mathrm{ABX}$-type in which nuclei $\mathrm{A}$ and $\mathrm{B}$ undergo intramolecular exchange, the $\mathrm{AB}$ part may in the limit of slow exchange be decomposed into two AB-type quartets. Ganter, Sanford and Roberts ${ }^{8}$ have made the assumption that in ABX-type spin systems exchange of $A$ and $B$ may be treated as exchange in two superimposed AB-spin systems and employed the $A B$ equations derived by Alexander to evaluate the exchange rate from the lineshape of the $\mathrm{AB}$ part. The present computer programme allows a direct comparison of the simplified $A B$ treatment with the exact $\mathrm{ABK}$ or $\mathrm{ABX}$ treatment. As perhaps could be expected the use of Alexander's equations for the AB part results in lineshapes in close agreement with the exact ABX treatment when $\left|\nu_{\mathrm{A}}-\nu_{\mathrm{X}}\right|$ and $\left|\nu_{\mathrm{B}}-\nu_{\mathrm{X}}\right| \gg J_{\mathrm{AX}} \simeq J_{\mathrm{BX}}$

C) Exchange in four-spin systems. Two important cases of chemical exchange in four-spin systems may be treated by our present computer programme. One of these is the $\mathrm{AA}^{\prime} \mathrm{BB}^{\prime} \rightarrow \mathrm{A}_{4}$ case which is encountered experimentally for example in $\mathrm{N}$-alkylsubstituted aziridines (I) as was mentioned in the introduction
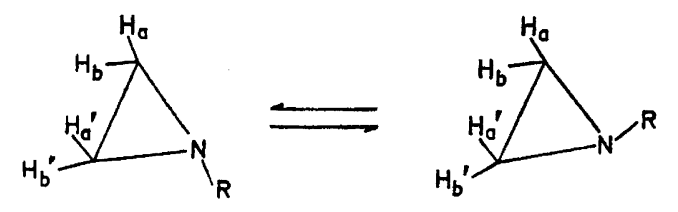

(interfering ${ }^{14} \mathrm{~N}$ quadrupole broadenings and spin couplings to the $R$ substituent may be removed by double irradiation). At slow rates of inversion the proton resonance spectrum is of the $\mathrm{AA}^{\prime} \mathrm{BB}^{\prime}$-type, but at high inversion rates all ring protons become effectively equivalent and their spectrum consists of a single line. A set of spectra calculated at different characteristic life times for this type of intramolecular exchange processes is shown in Fig. 3. The spectra are symmetric with respect to $\left(v_{\mathrm{A}}+\nu_{\mathrm{B}}\right) / 2$ at all $\tau$-values. Sample calculations show that the coalescence point, if defined as the temperature at which the 


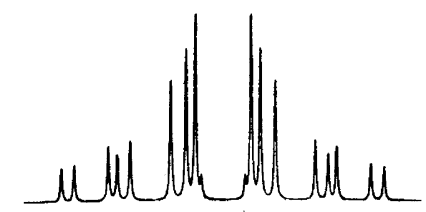

$\tau=100.0 \mathrm{sec}$

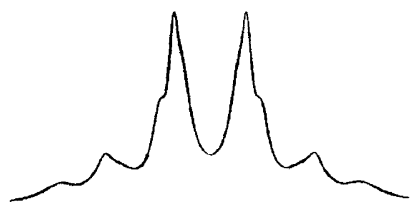

$\tau=0.0800 \mathrm{sec}$
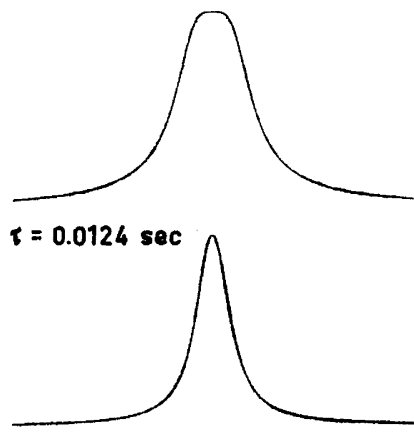

$\tau=0.0060 \mathrm{sec}$

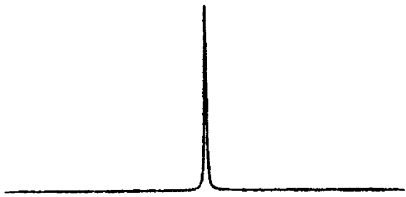

$\gamma=10^{-6} \mathrm{sec}$

Fig. 3. Examples of theoretically calculated four-spin spectra of $\mathrm{AA}^{\prime} \mathrm{BB}^{\prime}$-type which are converted to an $A_{4}$ spectrum by fast interchange between $A$ and $B ; A^{\prime}$ and $B^{\prime}$. The spectra have been calculated with $v_{\mathrm{A}}=v_{\mathrm{A}}^{\prime}=0.0 \quad \mathrm{~Hz} ; \quad v_{\mathrm{B}}=v_{\mathrm{B}}{ }^{\prime}=25.0 \quad \mathrm{~Hz}$; $J_{\mathrm{AA}}{ }^{\prime}=J_{\mathrm{BB}^{\prime}}=15.0 \mathrm{~Hz} ; J_{\mathrm{AB}}=10.0 \mathrm{~Hz} ;$
$J_{\mathrm{AB}}=J_{\mathrm{AB}}=5.0 \mathrm{~Hz}$, and $T_{2}=1.0$ sec.
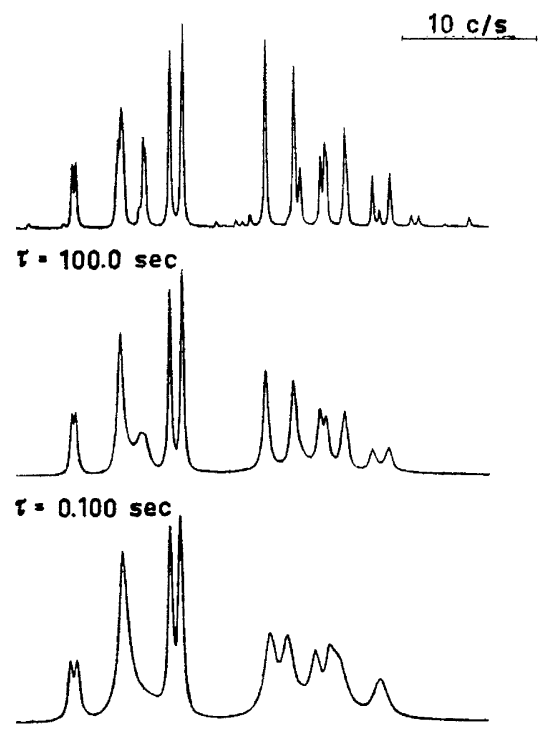

$r=0.020 \mathrm{sec}$
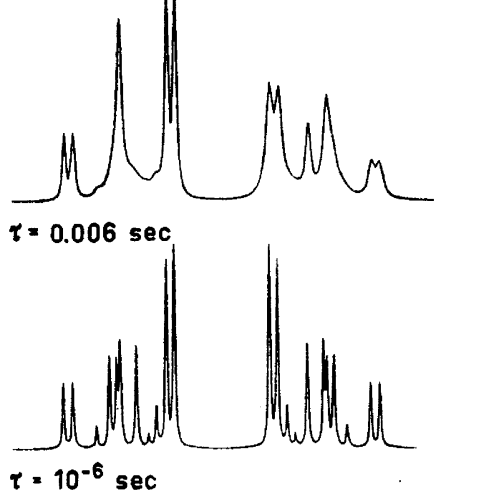

Fig. 4. Examples of theoretically calculated four-spin spectra of ABCD-type which are converted to an $A_{2} B_{2}$ spectrum by fast exchange between $A$ and $B ; C$ and $D$. The spectra have been calculated with $v_{\mathrm{A}}=0.0 \mathrm{~Hz} ; \nu_{\mathrm{B}}=5.0 \mathrm{~Hz} ; \nu_{\mathrm{C}}=25.0 \mathrm{~Hz}$; $\nu_{\mathrm{D}}=35.0 \mathrm{~Hz} ; J_{\mathrm{AB}}=15.0 \mathrm{~Hz} ; J_{\mathrm{AC}}=10.0 \mathrm{~Hz}$; $J_{\mathrm{AD}}=5.0 \mathrm{~Hz} ; J_{\mathrm{BD}}=10.0 \mathrm{~Hz}: J_{\mathrm{CD}}=12.0 \mathrm{~Hz}$, and $T_{2}=1.0$ sec.

two halves of the spectrum have merged into one broad singlet with no dip in the center, is obtained for $\tau=0.0124$ sec with the parameters used in Fig. 3.

An approximate value for the mean life time $\tau_{\mathrm{c}}$ at the coalescence point may be obtained through the equation

Acta Chem. Scand. 24 (1970) No. 2 


$$
\tau_{\mathrm{C}}=\frac{\sqrt{2}}{2 \pi\left(\nu_{\mathrm{A}}-\nu_{\mathrm{B}}\right)}
$$

which is valid for the coalescence of two singlets of equal intensity under the assumption that $1 / T_{2}=0$. With $\nu_{\mathrm{A}}-\nu_{\mathrm{B}}$ equal to $25 \mathrm{~Hz}$ eqn. (15) predicts a $\tau_{\mathrm{C}}$-value of $0.00901 \mathrm{sec}$ or a factor 1.4 lower than the "true" value given above.

If the exchange rate at the coalescence is estimated from eqn. (15) the resulting value of $\Delta G^{\neq}$will in the case discussed above be lower than the correct value by about $0.2 \mathrm{kcal} / \mathrm{mol}$ at $25^{\circ} \mathrm{C}$. This accuracy may be considered acceptable in a preliminary investigation. It seems likely that the errors introduced in the parameters when eqn. (15) is applied should decrease as the coupling constants in the $\mathrm{AA}^{\prime} \mathrm{BB}^{\prime}$ spin system (in particular $\mathrm{M}=J_{\mathrm{AA}}{ }^{\prime}-J_{\mathrm{BB}}{ }^{\prime}$ and $\mathrm{L}=J_{\mathrm{AB}}-J_{\mathrm{AB}}{ }^{\prime}$ ) decrease. Unfortunately, high computer costs at present preclude additional calculations to test this assumption.

The second type of four-spin system in which chemical exchange can be treated by our present computer programme is the $\mathrm{ABCD} \rightarrow \mathrm{A}_{2} \mathrm{~B}_{2}$ case. Examples of this type of system are found in para-substituted benzaldehydes in which the ring proton spectrum is of ABCD-type at slow rotation of the aldehyde group and of $\hat{A}_{2} B_{2}$-type at high rates of rotation for this group. $A$ set of spectra calculated at different interchange rates for the $A B C D \rightarrow A_{2} B_{2}$ case is shown in Fig. 4.

\section{DISCUSSION}

In the evaluation of intramolecular exchange rates from the lineshapes in NMR spectra, complexity of the spectra is not necessarily a disadvantage. On the contrary, a richness of spectral details may in fact allow increased accuracy in the derived exchange rates, especially in the slow and fast exchange limits. Experimental investigations which illustrate this are presented in the two subsequent papers.

The application of the density matrix theory to spin systems involving more than four nuclei is desirable. The difficulties involved in such an extension are mainly of a computational nature. The calculation of one point on the lineshape curve in a five-spin spectrum with our present programme would require about $4 \mathrm{sec}$ on an IBM 360/75 computer and the corresponding time for a six-spin case is estimated to be 50 sec. A complete spectrum consisting of 300 points will thus for a five-spin case require some $20 \mathrm{~min}$ and for a sixspin case about $4 \mathrm{~h}$ of computation time. In a kinetic investigation a large number of spectra must in general be calculated, and with present day computers treatment of six-spin problems seems completely out of the question. A somewhat unorthodox way of reducing the computer time required for a kinetic investigation is to first calculate theoretical spectra at different exchange rates using relevant spin coupling and chemical shift data and then try to find the temperatures where the experimental spectra exactly match the theoretical. This type of approach is presently under study in our laboratories. 


\section{REFERENCES}

1. Anet, F. A. L. and Ahmad, M. J. Am. Chem. Soc. 86 (1964) 119.

2. Binsch, G. Topics in Stereo Chemistry, Wiley, N. Y. 1968, Vol. 3, p. 97.

3. Anet, F. A. L. and Sandström, J. To be published.

4. Mallory, F. B., Manath, S. L. and Wood, C. S. J. Am. Chem. Soc. 87 (1965) 5433.

5. Anet, F. A. L. and Bourn, A. J. R. J. Am. Chem. Soc. 89 (1967) 760.

6. Anet, F. A. L. and Osyany, J. M. J. Am. Chem. Soc. 89 (1967) 352.

7. Boulton, A. J., Katritzky, A. R., Sewell, M. J. and Wallis, B. J. Chem. Soc. 1967914.

8. Ganter, C., Sanford, M. P. and Roberts, J. D. J. Am. Chem. Soc. 88 (1966) 4235.

9. Kaplan, J. J. Chem. Phys. 28 (1958) 278.

10. Kaplan, J. J. Chem. Phys. 29 (1958) 462.

11. Alexander, S. J. Chem. Phys. 37 (1962) 967.

12. Alexander, S. J. Chem. Phys. 37 (1962) 974.

13. Dahlquist, K.-I. and Forsén, S. J. Chem. Phys. 73 (1969) 4124.

14. Dahlquist, K.-I. and Forsén, S. J. Magnetic Resonance 2 (1970) 61.

15. Kurland, R. J., Rubin, M. B. and Wise, W. B. J. Chem. Phys. 40 (1964) 2426.

16. Sunners, B., Piette, L. H. and Schneider, W. G. Can. J. Chem. 38 (1960) 681.

Received July 11, 1969. 